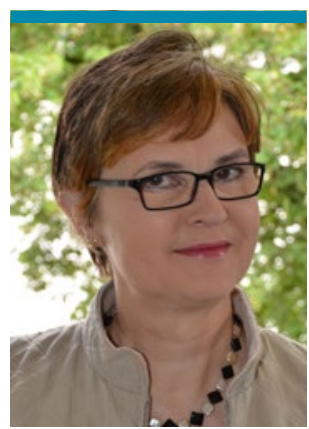

Prof. Dr. Franziska Ruëff, Klinik und Poliklinik für Dermatologie und Allergologie, Klinikum der Universität München

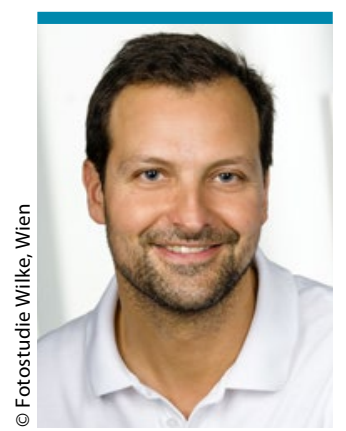

Prof. Dr. Gunter Sturm, Allergieambulatorium Reumannplatz, Wien; UniversitätsHautklinik Graz, Österreich

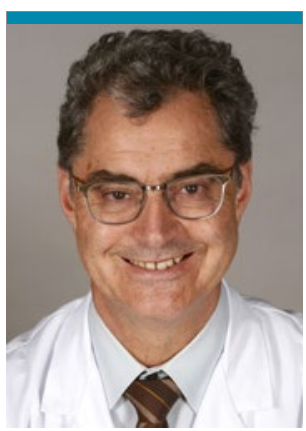

Prof. Dr. Peter SchmidGrendelmeier, Dermatologische Klinik und Poliklinik, Universitätsspital Zürich, Schweiz

\title{
Neue Daten in der Diskussion
}

Z um 11. Mal findet am 19. und 20. Juni das Arbeitsgespräch Insektengiftallergie statt - nun schon zum zweiten Mal in Fulda. Seit der ersten Tagung im Jahre 1986 treffen sich alle drei bis vier Jahre Allergologen aus Deutschland, Österreich und der Schweiz, um aktuelle Forschungsergebnisse auf dem Gebiet der Insektengiftallergie zu präsentieren und zu diskutieren. Auch dieses Mal ist es gelungen, renommierte Arbeitsgruppen zur Einsendung interessanter Beiträge zu motivieren und ein vielseitiges Treffen zu organisieren.

Verschiedene epidemiologische Untersuchungen beschäftigen sich mit Häufigkeit und Risikofaktoren der Insektengiftallergie. Ganz allgemein ist die Prävalenz anaphylaktischer Reaktionen besonders während der warmen Jahreszeit erhöht, was auf die während der Insektenflugzeit erfolgenden Stichreaktionen zurückzuführen sein dürfte (W. Sieber et al.). Besonders gefährdet für schwere Reaktionen auf Insektenstiche erwiesen sich nach der Auswertung von Daten aus dem Anaphylaxieregister - Patienten in höherem Lebensalter, mit Mastozytose und mit bestimmten kardiovaskulären Medikamenten (W. Francuzik et al). Weiter zeigt sich, dass betroffene Patienten oft keine adäquate langfristige Versorgung erhalten (M. Manmohan et al).

Was die hierzulande ursächlichen Insekten anbetrifft, so legen Einzelfallbeobachtungen nahe, dass auch als friedfertig geltende Langkopfwespen Stichreaktio-

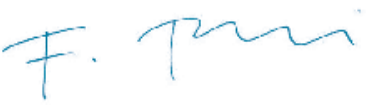

nen verursachen können (J. K. Tietze) und es nicht trivial ist, diese von Kurzkopfwesen zu unterschieden (V. Mauss).

Eine weitere Studie beschäftigt sich mit dem Hautpricktest und zeigte eine deutlich erhöhte Sensitivität bei Verwendung einer Konzentration von $300 \mu \mathrm{g} / \mathrm{ml}$, was allerdings mit Einbußen bei der Spezifität erkauft wird (D. Wiedemann).

Mehrere Arbeitsgruppen beschäftigen sich mit der komponentenbasierten Diagnostik. Diese kann zur Untersuchung von Insektengiftextrakten auf das Vorhandensein relevanter allergener Giftbestandteile benutzt werden (S. Blank et al.), neue oder bekannte kreuzreagierende Giftbestandteile erkennen (M. Schiener et al.) und auch zur Unterscheidung von Doppelsensibilisierungen (L. Arzt et al.) dienen.

Weiter im Fokus steht der prädiktive Wert eines mit verschiedenen Methoden erzielten Nachweises einer Sensibilisierung: Ein unauffälliger BAT schloss in einer Pilotstudie zwar schwere Reaktionen bei Stichprovokation aus, leichtere Systemreaktionen dagegen nicht (D. Bokanovic et al). Auch während und nach SIT mit Hymenopterengift (HG-SIT) ändern sich viele Parameter, ohne dass eine klare Prognose daraus ersichtlich ist (C. Möbs et al).

Mittlerweile ist gesichert, dass Mastzellerkrankungen ein Risiko bei Insektengiftallergie sind. Erneut wurde ein tödlicher Ausgang bei Feldstich in einer Kohorte von Patienten mit Mastozytose und

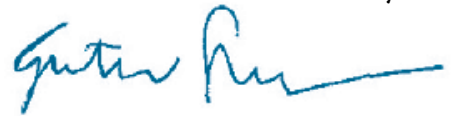

\begin{tabular}{|c|c|}
\hline \multicolumn{2}{|l|}{ Inhaltsverzeichnis } \\
\hline Thema & Seite \\
\hline Epidemiology and care research & 53 \\
\hline Diagnostics & 56 \\
\hline Mast cell diseases & 58 \\
\hline $\begin{array}{l}\text { Safety and efficacy of venom } \\
\text { immunotherapy }\end{array}$ & 60 \\
\hline \multicolumn{2}{|l|}{ Danksagung } \\
\hline \multicolumn{2}{|c|}{$\begin{array}{l}\text { Die Veranstalter danken der ALK-Abelló Arznei- } \\
\text { mittel GmbH, Hamburg, für die Unterstützung } \\
\text { des 11. Arbeitsgesprächs Insektengiftallergie. }\end{array}$} \\
\hline
\end{tabular}

Insektengiftallergie beobachtet, sogar trotz HG-SIT (R. Treudler et al).

Die HG-SIT ist gut verträglich, wobei eine größere Fallserie ergab, dass systemische Reaktionen eher bei längerer Einleitung auftraten (J. Stoevesandt). Eine Fallserie an Patienten mit malignen Tumoren erbrachte, dass auch bei malignen Grunderkrankungen eine HG-SIT sicher und wirksam ist (J. Aeberhard). Wenn allerdings bei einzelnen Patienten systemische Reaktionen auftreten, die die Fortsetzung der Therapie gefährden, so konnten mehrere Fallberichte die Effektivität einer Vorbehandlung mit einem AntiIgE-Antikörper zeigen (D. Wieczorek et al., R. Thonke et al., K. Berndt et al). Leider ist eine Studie dazu auch weiter nicht in Sicht!

Wir hoffen, dass Sie bei der Lektüre der Abstracts neue Erkenntnisse gewinnen und Anregungen für Ihren klinischen Alltag mitnehmen können!

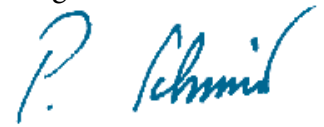

